\title{
Evaluation of Absorption Kinetics of Orally Administered Theophylline in Rats Based on Gastrointestinal Transit Monitoring by Gamma Scintigraphy
}

\author{
SHUNJI HARUTA, ${ }^{1,2}$ KEIICHI KAWAI, ${ }^{3}$ SEISHI JINNOUCHI, ${ }^{4}$ KEN-ICHI OGAWARA, ${ }^{2}$ KAZUTAKA HIGAKI, ${ }^{2}$ \\ SHOZO TAMURA, ${ }^{4}$ KAZUHIKO ARIMORI, $^{1}$ TOSHIKIRO KIMURA $^{2}$ \\ ${ }^{1}$ Department of Hospital Pharmacy, Central Research Laboratories, and Department of Radiology, Miyazaki Medical College, \\ 5200 Kihara, Kiyotake-cho, Miyazaki 889-1692, Japan \\ ${ }^{2}$ Department of Pharmaceutics, Faculty of Pharmaceutical Sciences, Okayama University, 1-1-1 Tsushima-naka, Okayama \\ 700-8530, Japan \\ ${ }^{3}$ Central Research Laboratories, Miyazaki Medical College, 5200 Kihara, Kiyotake-cho, Miyazaki 889-1692, Japan \\ ${ }^{4}$ Department of Radiology, Miyazaki Medical College, 5200 Kihara, Kiyotake-cho, Miyazaki 889-1692, Japan
}

Received 31 Jaunary 2000; revised 28 September 2000; accepted 6 October 2000

\begin{abstract}
The gastrointestinal (GI) transit and absorption of orally administered theophylline, a highly absorbable drug without presystemic elimination, were investigated under fasted and fed conditions using three rats in a crossover study. To evaluate the GI transit rate for each segment in vivo, a noninvasive technique, gamma scintigraphy, was employed using a nonabsorbable compound, ${ }^{99 \mathrm{~m}} \mathrm{Tc}$-labeled diethylenetriamine pentaacetic acid (DTPA). Using a gamma scintigraphic technique it is possible to simultaneously evaluate the GI transit and absorption of orally administered drug in the same individual. Theophylline was simultaneously administered along with [ ${ }^{99 m}$ Tc]DTPA to animals in the fasted and fed states. Each GI transit pattern, simulated using the GI transit-kinetic model with a lag time factor, was well fitted to the experimental data. Gastric emptying rate varied in each study, even under the same experimental condition. The GI transit pattern for each segment was highly variable, especially in animals in the fed state. This inconsistency in transit pattern was mainly due to the variability in gastric emptying, which was much slower in animals in the fed compared with the fasted state. However, in spite of a large variability of GI transit kinetics, the plasma concentration-time curves of theophylline were well predicted by the GI transit-absorption model using the individual GI transit parameters obtained in the study. The absorption rate of theophylline was considerably reduced in animals in the fed state, because of the reduction of gastric emptying rate. Analysis using GI transit-absorption model and gamma scintigraphic technique made it possible to estimate the variable absorption kinetics regulated by GI transit with huge variability. ( 2001 Wiley-Liss, Inc. and the American Pharmaceutical Association J Pharm Sci 90:464-473, 2001
\end{abstract}

Keywords: oral absorption kinetics; gastrointestinal transit; gamma scintigraphy; GI transit absorption model; theophylline

Correspondence to: T. Kimura (Telephone: 81-86-251-7948; Fax: 81-86-251-7926;

E-mail: kimura@pheasant.pharm.okayama-u.ac.jp)

Journal of Pharmaceutical Sciences, Vol. 90, 464-473 (2001)

(C) 2001 Wiley-Liss, Inc. and the American Pharmaceutical Association

\section{INTRODUCTION}

The gastrointestinal (GI) absorption of orally administered drugs is determined by two major factors, the permeability of GI mucosa and the GI 
transit rate. In particular, the GI transit rate is frequently affected by various factors (e.g., individual difference, ${ }^{1}$ meals, ${ }^{2}$ disease states, ${ }^{3}$ etc.), resulting in remarkable alteration of the absorption profile of orally administered drugs.

In our previous paper, ${ }^{4}$ a novel method based on GI transit kinetics was developed for estimating the absorption profiles of drugs administered orally as an aqueous solution. The validity and the utility of the prediction method were demonstrated for model drugs with different absorption characteristics in rats under the fasted condition, using the GI transit rate constant for each segment along the GI tract that was obtained as averaged values using phenol red as a nonabsorbable marker. However, to evaluate the drug absorption kinetics, especially in animals in the fed condition, the prediction of the plasma concentration-time curve after oral administration should be performed using each individual transit kinetics, because GI transit kinetics including gastric emptying could show a large intraindividual and/or interindividual variability in animals in the fed state. ${ }^{5,6}$

In the present study, a noninvasive technique, gamma scintigraphy, was used to evaluate the GI transit of orally administered drugs in rats. The use of gamma scintigraphy to assess GI transit in experimental and clinical practice has been well established and utilized. ${ }^{7-14}$ Even in rats ${ }^{15}$ and cats ${ }^{16}$ this method has already been applied to evaluate the gastric emptying profile. This technique allows simultaneous evaluation of $\mathrm{G}$ transit and oral absorbability of drug in the same individual. As an absorbable drug without first-pass elimination after oral administration, ${ }^{17}$ theophylline was administered along with ${ }^{99 m}$ Tc-labeled diethylenetriamine pentaacetic acid $\left(\left[{ }^{99 \mathrm{~m}} \mathrm{Tc}\right] \mathrm{DTPA}\right)$ solution. The prediction of plasma concentrationtime curves for theophylline in each individual was performed using both the GI transit rate constant determined for each individual and the averaged absorption rate constant for each segment. The predicted plasma concentration-time curve was compared with the observed data in the same individual, and the effect of food on the absorption kinetics was also investigated.

\section{EXPERIMENTAL SECTION}

\section{Materials}

Aminophylline (Sigma Chemical Company, St. Louis, MO) and theophylline (Tokyo Kasei Kogyo
Company, Tokyo, Japan) were obtained commercially. All chemicals and reagents were analytical grade commercial products. The ${ }^{99 \mathrm{~m}} \mathrm{Tc}$ was obtained as sodium pertechnetate solution by elution of a generator, and [ $\left.{ }^{99 \mathrm{~m}} \mathrm{Tc}\right] \mathrm{DTPA}$ was prepared using a kit containing calcium trisodium diethylenetriamine pentaacetate and stannous chloride dihydrate (Daiichi Radioisotope Lab., Tokyo, Japan).

\section{Animals}

Three male Wistar rats weighing 400-500 g (Kyudo Farm, Fukuoka, Japan) were fasted for $24 \mathrm{~h}$ prior to and during the experiment in the fasted study. In the case of the fed study, after fasting for $24 \mathrm{~h}$, rats were allowed free access to standard laboratory chow (Clea Japan, Tokyo, Japan) for $12 \mathrm{~h}$ just before starting the study. Our investigation was performed after approval by our local ethical committee at Miyazaki Medical College and in accordance with "Interdisciplinary Principles and Guidelines of the Use of Animals in Research".

\section{Gamma Scintigraphic Study}

A gamma scintigraphy, noninvasive technique was employed to measure GI transit rate for each segment with a gamma camera system (PRISM 3000-Odyssay, Picker International Inc., Cleveland, OH). An aqueous solution of [ $\left.{ }^{99 \mathrm{~m}} \mathrm{Tc}\right] \mathrm{DTPA}$, a nonabsorbable compound, ${ }^{8}$ was prepared as a tracer passing through GI tract. [ ${ }^{99 \mathrm{~m}}$ Tc]DTPA $(0.4-0.5 \mathrm{mCi} /$ body) was administered intragastrically to the rat using a gastric sonde with an absorbable drug. The dosed rat was kept in a restraining cage that was placed on the scintillation camera. The gamma scintigraphic imaging started just after dosing and was carried out for $6 \mathrm{~h}$ at 2 -min intervals under the dynamic planar condition.

\section{Determination of GI Transit Rate Constant for Each Segment}

GI tract was divided into four segments: stomach (s), jejunum (jej), ileum (ile), cecum and below (ce). The region of interest (ROI) for each segment was determined as follows. The ROIs of stomach and cecum were determined by the scintigraphic images taken just after dosing and at the end of the experiments, respectively, because those images showed almost the entire GI segment containing almost all the dosed [ $\left.{ }^{99 \mathrm{~m}} \mathrm{Tc}\right] \mathrm{DTPA}$. The small intestine could be basically separated to the 
proximal half and distal half by drawing a horizontal line, which we defined as jejunal and ileal segments, respectively. However, because the demarcation for both segments is not clear, some corrections were needed based on close examination of the [ $\left.{ }^{99 \mathrm{~m}} \mathrm{Tc}\right] \mathrm{DTPA}$ locus using 120 images taken during each whole study, as reported by Digenis et al. ${ }^{13}$ The important point for those corrections is that the locus and transit direction of $\left[{ }^{99 \mathrm{~m}} \mathrm{Tc}\right] \mathrm{DTPA}$ must be closely examined throughout the study and then the position of the segment can be judged. The radioactivity in each segment was then measured in each ROI, and the images were analyzed by computer-aided densitometry using the public domain National Institute of Health (NIH) Image program (http:// rsb. info.nih.gov/nihimage/). After correcting the decay of gamma ray by using the half-life of ${ }^{99 \mathrm{~m}} \mathrm{Tc}(6.02 \mathrm{~h})$, the relative percent of radioactivity in each ROI was plotted against time. The GI transit rate constant $\left(k_{i}\right)$ of each segment $i$ for each individual study was obtained by fitting the experimental GI transit data to the GI transitkinetic model $^{4}$ with the nonlinear regression program MULTI (FILT). ${ }^{18}$ In the present study, a lagtime factor in the ileocecal region was introduced into the GI transit-kinetic model.

\section{Determination of Absorption Rate Constant for Each Segment}

The absorption experiments were performed for the jejunum and ileum by a conventional in situ closed-loop method. ${ }^{19}$ The initial concentration of aminophylline and the period of absorption experiment were $0.5 \mathrm{mg} / \mathrm{mL}$ and $5 \mathrm{~min}$, respectively. The first-order absorption rate constant $\left(k_{\mathrm{a}}\right)$ was estimated by the rate of disappearance of drug from the lumen. The averaged $k_{\mathrm{a}_{i}}$ values of theophylline thus obtained for the jejunum and ileum, which were cited in our previous paper, ${ }^{4}$ are 11.882 and $8.080 \mathrm{~h}^{-1}$, respectively. The values of $k_{\mathrm{a}}$ of stomach and colon were assumed to be zero because no contribution from these segments to the absorption of theophylline was shown in our previous studies. ${ }^{4,20}$

\section{Oral and Intravenous Administration}

For the oral administration study, the right femoral artery was cannulated with vinyl tubing (i.d., $0.5 \times 0.8 \mathrm{~mm}$; Dural Plastics and Engineering, Australia) while the animal was under ether anesthesia. The solution of aminophylline $(5 \mathrm{mg} / 5$
$\mathrm{mL} / \mathrm{kg}$ ) was administered intragastrically to the rat along with a GI transit marker, $\left[{ }^{99 m}\right.$ Tc]DTPA. Blood samples were periodically taken from the cannulated femoral artery. The oral administration studies were performed with animals in the fasted condition (fasted for $24 \mathrm{~h}$ prior to and during the experiment, but allowed free access to water) and the fed condition after a recovery period of 1 week in each rat.

For the intravenous (iv) administration study, aminophylline was administered as a solution by bolus injection $(5 \mathrm{mg} / 5 \mathrm{~mL} / \mathrm{kg}$ ) into the left femoral vein, and blood samples were periodically taken from the cannulated right femoral artery. The plasma concentration-time curve of theophylline was analyzed by a two-compartment model. The pharmacokinetic parameters were estimated by fitting the plasma concentration versus time data after iv administration to a model equation $C_{\mathrm{p}}=A \mathrm{e}^{-\alpha t}+B \mathrm{e}^{-\beta t}$.

\section{Analytical Method}

Theophylline in the plasma was determined by the reversed-phase high-performance liquid chromatography (HPLC) method. ${ }^{21}$ Briefly, plasma samples were deproteinized with acetonitrile. After centrifugation at $3000 \mathrm{rpm}$ for $10 \mathrm{~min}$, the supernatant of the mixture was injected onto the HPLC system, which consisted of a high-performance liquid chromatograph (L-7100, Hitachi, Tokyo) connected to an ODS column (SPHERISORB ODS-2-5 $(250 \times 4.6 \mathrm{~mm})$ Chemco Scientific Company, Tokyo) and an ultraviolet (UV) detector (L-7400, Hitachi). The mobile phase, flow rate, and wavelength of the detector were $5 \mathrm{mM}$ acetate buffer ( $\mathrm{pH} 4.8$ ): acetonitrile (9:1 by volume), 1.0 $\mathrm{mL} / \mathrm{min}$, and $272 \mathrm{~nm}$, respectively.

\section{Statistical Analysis}

A student's paired $t$ test was performed to estimate the significant difference between the fed and fasted conditions. Statistical significance of the correlation between observed and calculated values of plasma concentration were determined by Pearson's method, which is a method to estimate the significance for the linear correlation by calculating Pearson's correlation coefficient.

\section{THEORETICAL SECTION}

The GI transit-absorption model incorporating a lagtime factor in ileocecal region is shown in Figure 1. Although we defined it as eight segments 


\section{Gl-Transit Kinetic Model}

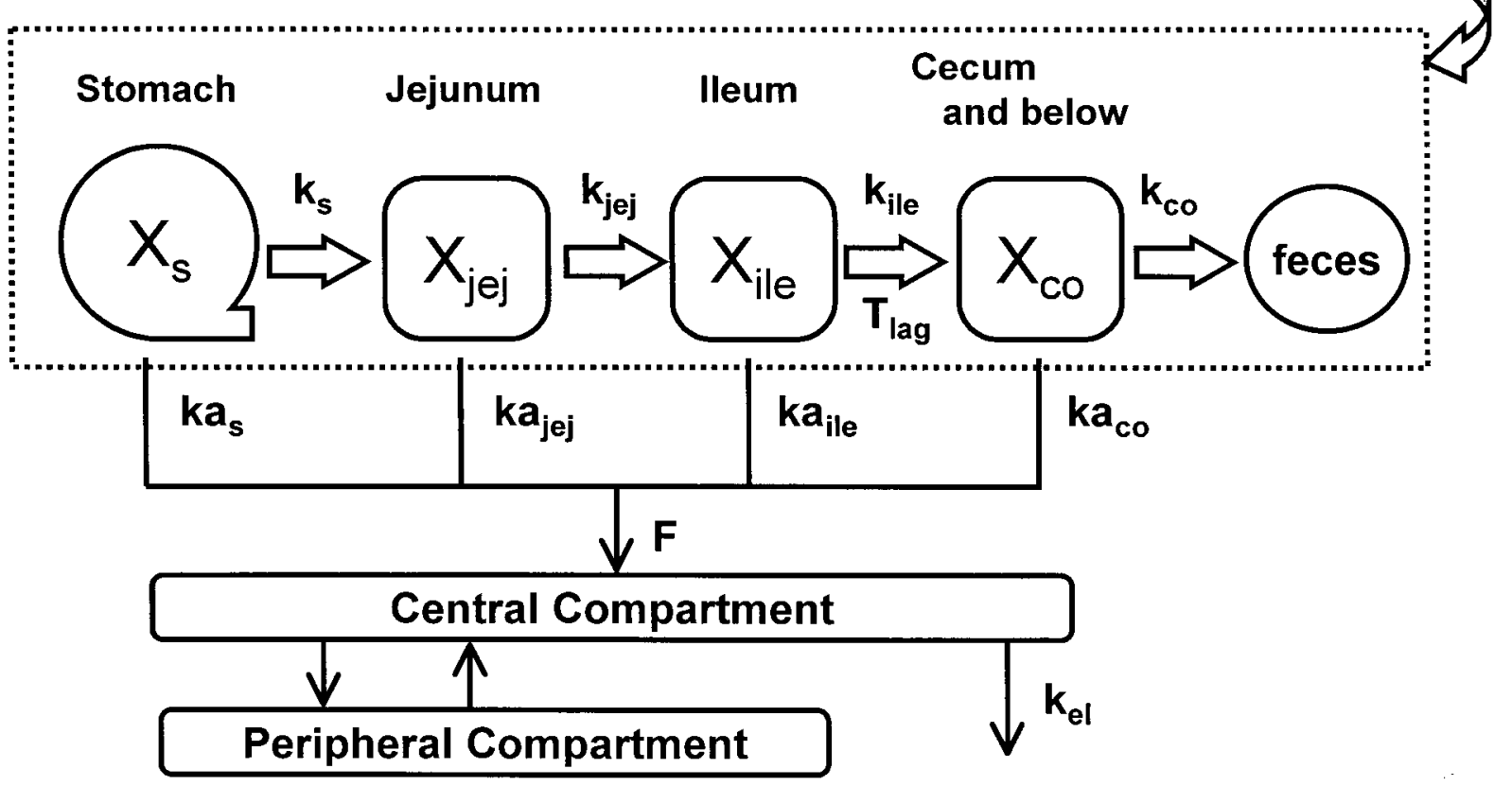

Figure 1. The GI transit-absorption model is a pharmacokinetic model containing a GI transit and an absorption process in each segment. Key: (D) initially administered dose; $\left(X_{i}\right)$ amount of drug in the segment $i$; $\left(k_{i}\right)$ first-order transit rate constant from the segment $i ;\left(k_{a_{i}}\right)$ first-order absorption rate constant for the segment $i$; $\left(T_{\text {lag }}\right)$ onset time to transit from the ileum to cecum; $\left(k_{\mathrm{el}}\right)$ first-order elimination rate constant from central compartment; $(F)$ bioavailability without first-pass elimination.

in the previous studies, ${ }^{4,20}$ gastrointestinal tract was divided into four segments because it was difficult to distinguish the upper part from the lower of jejunum or ileum by gamma scintigraphic images. An absorbable drug transits from a segment to the next segment with segmental absorption (first-order absorption). Gastric emptying rate and transit rate for each segment are represented by eqs. 1-4, by substituting zero for $k_{\mathrm{a}_{i}}$ in the case of a nonabsorbable drug like $\left[{ }^{99 m}\right.$ Tc]DTPA, which corresponds to the GI transit-kinetic model.

$$
\frac{\mathrm{d} X_{\mathrm{s}}}{\mathrm{d} t}=-\left(k_{\mathrm{s}}+k_{\mathrm{a}_{\mathrm{s}}}\right) \cdot X_{\mathrm{s}}
$$

where at $t=0, X_{s}=D_{\mathrm{po}}$ (the dose of the orally administered drug).

$$
\begin{aligned}
\frac{\mathrm{d} X_{\mathrm{jej}}}{\mathrm{d} t} & =k_{\mathrm{s}} \cdot X_{\mathrm{s}}-\left(k_{\mathrm{jej}}+k_{\mathrm{ajej}}\right) \cdot X_{\mathrm{jej}} \\
\frac{\mathrm{d} X_{\mathrm{ile}}}{\mathrm{d} t} & =k_{\mathrm{jej}} \cdot X_{\mathrm{jej}}-k_{\mathrm{aile}} \cdot X_{\mathrm{ile}} \quad\left(0 \leq t \leq T_{\mathrm{lag}}\right) \\
& =k_{\mathrm{jej}} \cdot X_{\mathrm{jej}}-\left(k_{\mathrm{ile}}+k_{\mathrm{aile}}\right) \cdot X_{\mathrm{ile}} \quad\left(t>T_{\mathrm{lag}}\right)
\end{aligned}
$$

$$
\frac{\mathrm{d} X_{\mathrm{ce}}}{\mathrm{d} t}=k_{\mathrm{ile}} \cdot X_{\mathrm{ile}}-\left(k_{\mathrm{co}}+k_{\mathrm{aco}_{\mathrm{co}}}\right) \cdot X_{\mathrm{co}} \quad\left(t>T_{\mathrm{lag}}\right)
$$

where $X, k$, and $k_{\mathrm{a}}$ represent the amount, the transit rate constant, and the absorption rate constant, respectively, $T_{\text {lag }}$ represents an onset time when the dug transits from the ileum to cecum in the ileocecal region, and the subscripts for parameters indicate each GI site.

The prediction of the plasma concentrationtime curve by the convolution method consists of four-step analyses as described in our previous paper. ${ }^{4}$ The predicted profile represents the plasma concentration-time profile without the first-pass elimination in intestinal epithelium and/or liver.

\section{RESULTS AND DISCUSSION}

The technique of gamma scintigraphy was introduced for the evaluation of oral dosage forms more than 20 years ago. ${ }^{7}$ Since then, the use of gamma scintigraphy has been well established and utilized to assess GI transit in experimental and 


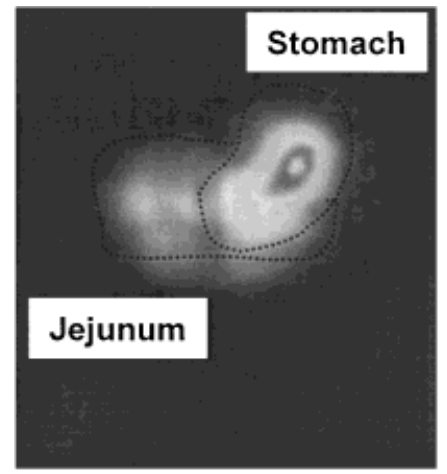

Scene 1

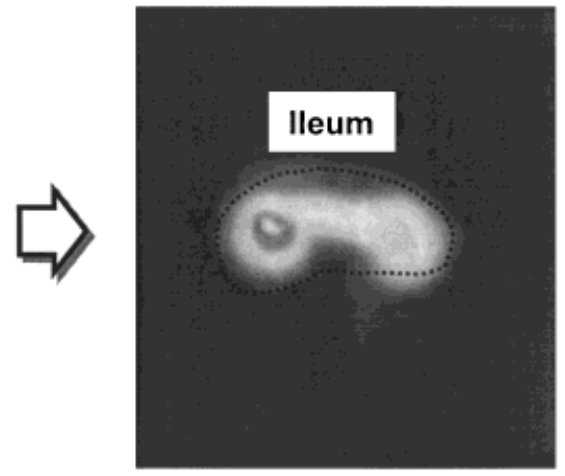

Scene 2

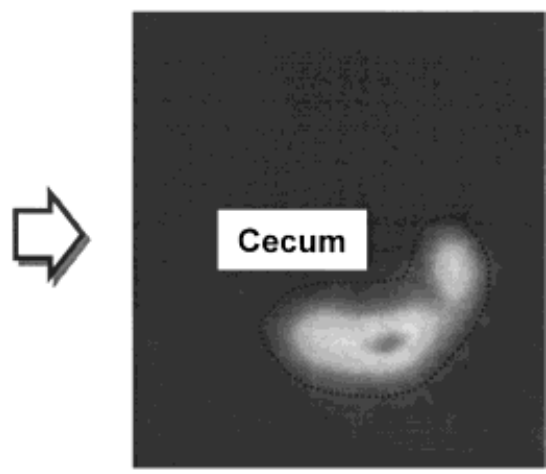

Scene 3

Figure 2. Typical scintigraphic images of orally administered [ $\left.{ }^{99 \mathrm{~m}} \mathrm{Tc}\right] \mathrm{DTPA}$ along the GI tract: (Scene 1) $7 \mathrm{~min}$; (Scene 2) $153 \mathrm{~min}$; (Scene 3) 295 min after oral administration.

clinical practice. ${ }^{8-16}$ In this study, we applied this technique to rats and examined the effect of GI transit rate on the absorption kinetics of orally administered aminophylline.

Figure 2 shows typical gamma scintigraphic images of $\left[{ }^{99 \mathrm{~m}} \mathrm{Tc}\right] \mathrm{DTPA}$ passing through the GI tract postdose. Orally administered [ $\left.{ }^{99 \mathrm{~m}} \mathrm{Tc}\right] \mathrm{DTPA}$ was confirmed to periodically migrate below along the GI tract. It is difficult to discriminate jejunum from ileum in the gamma scintigrapy images. However, as Digenis et al. reported, ${ }^{13}$ it was possible to subdivide the small intestine by closely examining the relative position of the radioactivity within the field of view of the gamma camera. Figure 3 shows both observed time courses of the relative percent of radioactivity in each segment determined by the gamma scintigraphic study and theoretical curves based on the GI transit-kinetic model in rats in each feeding condition. Because a lagtime is clearly observed in the transit process from the ileum to the cecum in every study, the lagtime was introduced in the GI transit-kinetic model. A similar lagtime was observed in a human study. ${ }^{13}$ The necessity of a lagtime means that it is difficult to describe the transit kinetics in ileum by a single well-stirred compartment. However, as is evident from the figure, the theoretical curve of radioactivity for each segment, which was obtained from the fitting analysis, was significantly in good agreement with the observed data in each individual study. This result indicates that the GI transit-absorption model, which consists of four GI segments with a lagtime factor in the ileocecal region, could be practically useful. The corresponding GI transit parameters are summarized in Table 1. GI transit rate constants for stomach $\left(k_{\mathrm{S}}\right)$ and jejunum $\left(k_{\mathrm{jej}}\right)$ were reasonable values by comparison with their corresponding $C L_{\mathrm{g}_{i}}$ values ${ }^{4}\left(C L_{\mathrm{g}_{i}}\right.$ represents the transit clearance in the segment $i$, calculated model independently with the equation: $C L_{\mathrm{g}_{i}}=100 / \mathrm{AUCRi}_{0-12 \mathrm{~h}}$, where AUCRi $_{0-12 \mathrm{~h}}$ indicates the area under the percent of dosed total radioactivity versus time curve from 0 to $12 \mathrm{~h}$ after dosing). However, in the case of ileum, larger values of $k_{\text {ile }}$ than $C L_{\mathrm{g}_{i}}$ of ileum were given in four of six cases because of introducing a lagtime factor into the ileocecal region, which means $C L_{\mathrm{g}_{i}}$ should be utilized to discuss the intestinal transit of drug in this study. Although the introduction of lagtime factor to the GI transit-absorption model sometimes resulted in overestimation of $k_{i}$ values, such a factor has been found to be quite useful for the description of the GI transit of drug after oral administration.

GI transit behavior was remarkably affected by the different food conditions. Under the fasted condition, gastric emptying of [ $\left.{ }^{99 \mathrm{~m}} \mathrm{Tc}\right] \mathrm{DTPA}$ was completed by $1 \mathrm{~h}$ postdose for all three rats examined, resulting in gastric emptying clearance $\left(C L_{\mathrm{g}_{\mathrm{s}}}\right)$ from 6.63 to $29.76 \mathrm{~h}^{-1}$. Therefore, almost the entire dosed marker reached the cecum within $4 \mathrm{~h}$. In contrast, under the fed condition $C L_{\mathrm{g}_{\mathrm{s}}}$ significantly decreased to 0.35 to $3.92 \mathrm{~h}^{-1}$ $\left(1.10 \pm 1.07 \mathrm{~h}^{-1}, p<0.05\right)$, and in two of three cases, the gastric emptying was not complete even $6 \mathrm{~h}$ postdose, resulting in the prolongation of the time for all the marker dosed to reach the cecum. On the other hand, the same tendency was found for the transit in jejunum and ileum under both food conditions, as can be seen in the values of $C L_{\mathrm{g}_{i}}$. These results support the reports regarding the effect of food on the GI transit of drugs in human; ${ }^{6,22-24}$ that is to say, the food would delay 

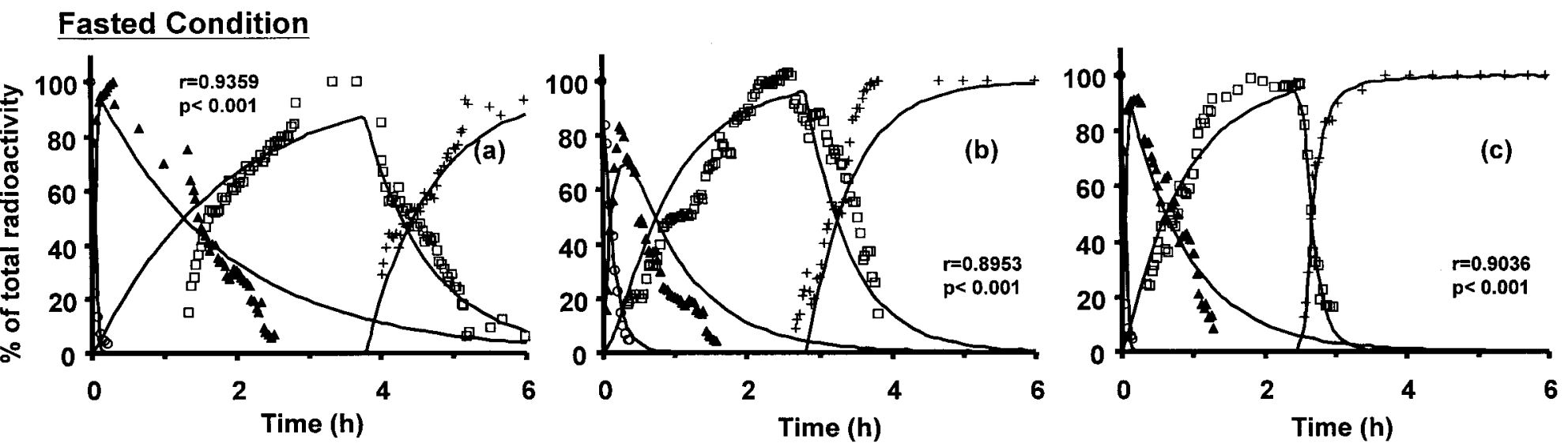

\section{Fed Condition}
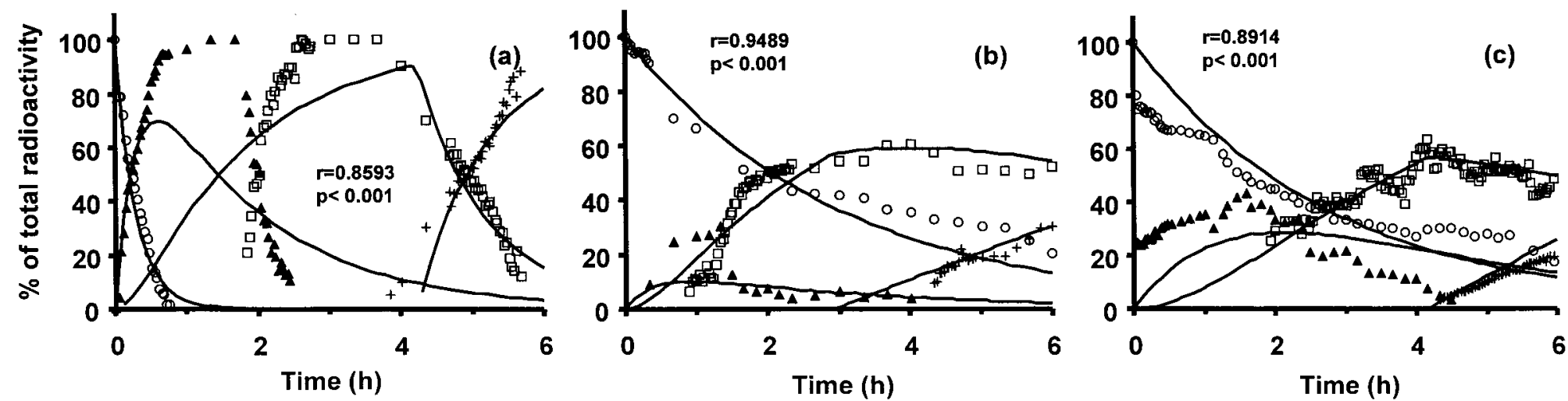

Figure 3. Time course of the remaining percent of dosed total radioactivity in each GI segment after oral administration of [ [9 $\mathrm{Tc}$ TCDTPA in rats. [ $\left.{ }^{99 \mathrm{~m}} \mathrm{Tc}\right]-D T P A$ solution was administered intragastrically. Experiments were performed for $3 \mathrm{rats}$ (a, b, and c) under fasted and fed conditions. Scintigraphic images were taken at 2-min intervals for $6 \mathrm{~h}$. Observed time courses of the remaining percent of dosed total radioactivity in each GI segment are expressed as follows: $(\bigcirc)$, stomach; ( $\mathbf{\Lambda})$, jejunum; $(\square)$ ileum; $(+)$, cecum and below. Calculated time courses of the percent of dosed total radioactivity in each GI segment are expressed by solid lines, using $k_{i}$ values obtained from the fitting by MULTI(FILT) program. Statistical significance of the correlation between observed and calculated values was examined by Pearson's method. 
Table 1. Gastrointestinal Transit Parameters for Each Segment

\begin{tabular}{|c|c|c|c|c|c|c|}
\hline \multirow[b]{2}{*}{ Food condition } & \multirow[b]{2}{*}{ Rat No. } & \multicolumn{4}{|c|}{ GI Transit Rate Constant $\left(\mathrm{h}^{-1}\right) / C L_{\mathrm{g}_{i}}\left(\mathrm{~h}^{-1}\right)^{a}$} & \multirow[b]{2}{*}{ Lagtime (h) } \\
\hline & & Stomach & Jejunum & Ileum & Cecum $^{c}$ and below & \\
\hline \multirow[t]{3}{*}{ Fasted } & $\mathrm{a}$ & $\begin{array}{c}31.171 / \\
29.76\end{array}$ & $\begin{array}{c}0.55 / \\
0.57\end{array}$ & $\begin{array}{c}1.23 / \\
0.30\end{array}$ & $\begin{array}{c}0.199 / \\
-b\end{array}$ & 3.79 \\
\hline & $\mathrm{b}$ & $\begin{array}{c}6.89 / \\
6.63\end{array}$ & $\begin{array}{c}1.19 / \\
1.28\end{array}$ & $\begin{array}{c}1.71 / \\
0.39\end{array}$ & $\begin{array}{c}0.404 / \\
-b\end{array}$ & 2.83 \\
\hline & $\mathrm{c}$ & $\begin{array}{c}28.75 / \\
25.91\end{array}$ & $\begin{array}{c}1.17 / \\
1.23\end{array}$ & $\begin{array}{c}5.98 / \\
0.45\end{array}$ & $\begin{array}{c}0.182 / \\
-b\end{array}$ & 2.54 \\
\hline Mean $\pm \mathrm{SD}$ & & $\begin{array}{c}22.27 \pm 13.37 / \\
20.77 \pm 12.39\end{array}$ & $\begin{array}{c}0.97 \pm 0.36 / \\
1.03 \pm 0.40\end{array}$ & $\begin{array}{c}2.97 \pm 2.61 / \\
0.38 \pm 0.08\end{array}$ & $\begin{array}{c}0.262 \pm 0.124 / \\
-b\end{array}$ & $3.05 \pm 0.65$ \\
\hline \multirow[t]{3}{*}{ Fed } & $\mathrm{a}$ & $\begin{array}{c}3.57 / \\
3.92\end{array}$ & $\begin{array}{c}0.60 / \\
0.60\end{array}$ & $\begin{array}{c}1.10 / \\
0.29\end{array}$ & $\begin{array}{c}0.056 / \\
-b\end{array}$ & 4.22 \\
\hline & $\mathrm{b}$ & $\begin{array}{c}0.34 / \\
0.35\end{array}$ & $\begin{array}{c}2.43 / \\
2.24\end{array}$ & $\begin{array}{c}0.17 / \\
0.27\end{array}$ & $\begin{array}{c}0.372 / \\
-b\end{array}$ & 2.93 \\
\hline & $\mathrm{c}$ & $\begin{array}{c}0.36 / \\
0.39\end{array}$ & $\begin{array}{c}0.59 / \\
0.59\end{array}$ & $\begin{array}{r}0.27 / \\
0.34\end{array}$ & $\begin{array}{c}0.043 / \\
-b\end{array}$ & 4.17 \\
\hline Mean $\pm \mathrm{SD}$ & & $\begin{array}{l}1.42 \pm 1.86 / \\
1.10 \pm 1.07^{d}\end{array}$ & $\begin{array}{c}1.21 \pm 1.06 / \\
1.74 \pm 0.97\end{array}$ & $\begin{array}{c}0.51 \pm 0.51 / \\
0.34 \pm 0.24\end{array}$ & $\begin{array}{c}0.157 \pm 0.186 / \\
{ }_{b}^{b}\end{array}$ & $3.18 \pm 1.80$ \\
\hline
\end{tabular}

The gastrointestinal transit rate constant for each segment and a lagtime in the ileocecal region were obtained from the fitting of the experimental data to GI transit-kinetic model by using MULTI (FILT) program.

${ }^{a} C L_{\mathrm{g}_{i}}$, the transit clearance in segment $i$, was calculated by $100 / \mathrm{AUCR}_{0-12 \mathrm{~h}}$, where $\mathrm{AUCR}_{0-12 \mathrm{~h}}$ is the area under the percent of dosed total radioactivity versus time curve for each segment calculated by the trapezoidal rule.

${ }^{b}$ Not calculated.

$c \times 10^{-3} \mathrm{~h}^{-1}$.

${ }^{d} p<0.05$, compared with fasted condition.

the gastric emptying but not the intestinal transit of drugs.

Larger and the smaller values of $C L_{\mathrm{g}_{i}}$ in stomach and jejunum, respectively, than those in our previous report ${ }^{4,20}$ were noted. Some possible explanations for this result are as follows: (1) different compartment models were employed; (2) rats were kept in the different types of the restraining cages; (3) different nonabsorbable markers were utilized; and (4) the parameters in the previous report were obtained from the averaged values, which include large interindividual variance, whereas in the present study, the parameter for each individual subject was used. Although we need to figure out which factor would influence predominantly GI transit of drug, it should be taken into consideration that GI transit might be changed easily by some factors, even under the fasted condition.

The plasma concentration-time curve of theophylline after oral administration of aminophylline was estimated for each rat using GI transit rate constants determined for each rat in the fasted and fed conditions, according to our prediction method. ${ }^{4}$ The results are shown in Figure 4, together with the observed data. The equation describing the plasma concentration of theophylline after iv administration of aminophylline, which was used as the weight function, is $C_{\mathrm{p}}=14.6 \mathrm{e}^{-26.2 t}+10.2 \mathrm{e}^{-0.31 t}$. The predicted curves were significantly in good agreement with observed data in all the individual studies. As shown in Table 2, $T_{\max }$ and $C_{\mathrm{p}_{\max }}$ for theophylline, which were well predicted by the GI transitabsorption model, were remarkably influenced by the presence of food, although the difference in $T_{\max }$ was not significant because of large interindividual variability. This effect on the plasma concentration-time curve is caused by the alteration of GI transit behavior, mainly gastric emptying. In our previous study using rats with GI transit rate retarded by propantheline, ${ }^{20}$ we reported that changes in the absorption profiles could reflect the retarded gastric emptying rate, because theophylline is rapidly absorbed and the absorbability is not so different among the segments of the small intestine. The delay of gastric emptying by ingested foods was also reported to cause the delay of the absorption of several drugs, including theophylline in human, ${ }^{6,23}$ which coincided with our present result about the relationship among food, gastric emptying and 
Fasted Condition
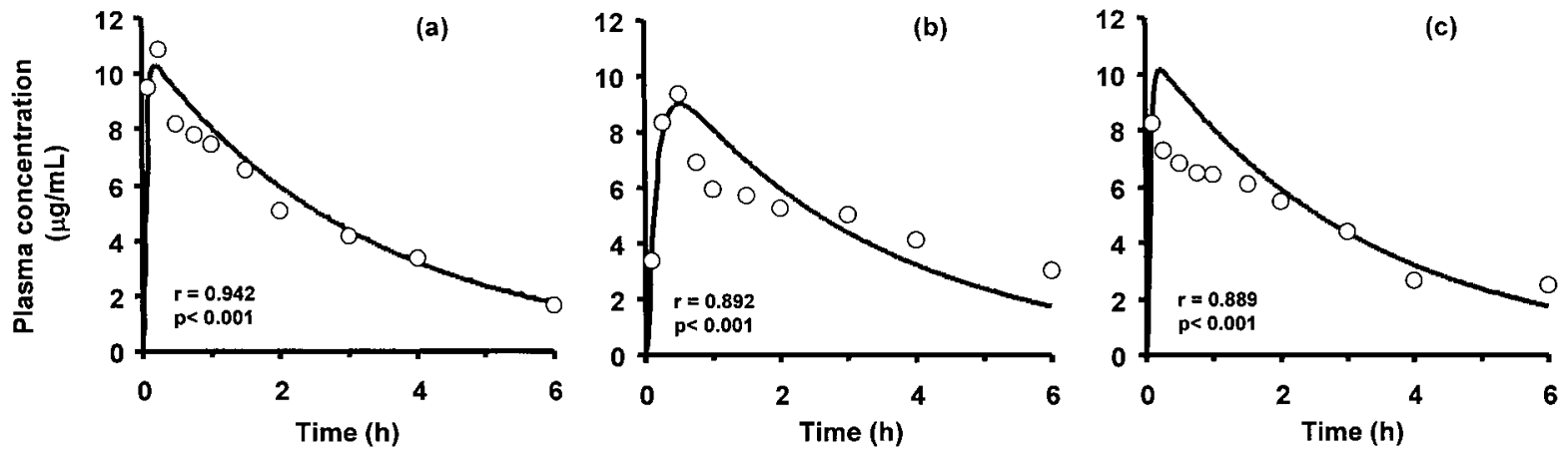

Fed Condition
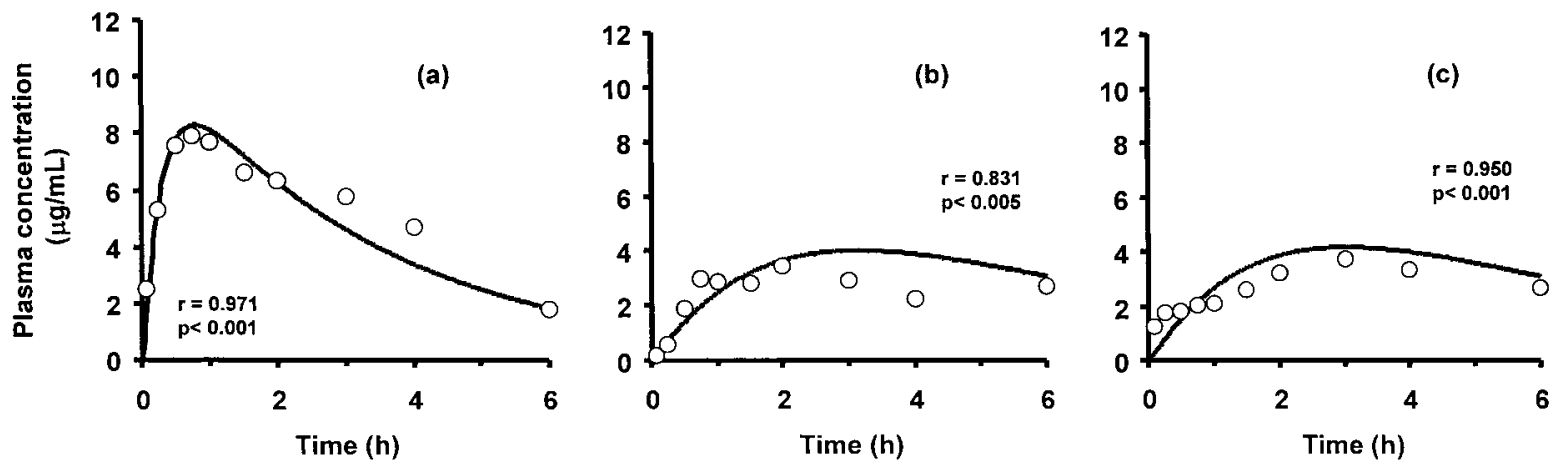

Figure 4. Plasma concentration-time curves of theophylline after oral administration of aminophylline in three rats (a, b, and c) under fasted and fed conditions. Aminophylline was administered at the dose of $5 \mathrm{mg} / \mathrm{kg}$. Results are expressed with an open circle for each experiment. Each predicted plasma concentration-time profile is expressed by the solid line, using the individual GI transit data obtained by the fitting analysis. Statistical significance of the correlation between observed and calculated values was examined by Pearson's method.

Table 2. Comparison of Pharmacokinetic Parameters for Theophylline Predicted by GI Transit-Absorption Model with those from Observed Data

\begin{tabular}{|c|c|c|c|c|}
\hline \multirow[b]{2}{*}{ Food Condition } & \multirow[b]{2}{*}{ Rat No. } & \multicolumn{3}{|c|}{ Observed/Predicted ${ }^{a}$} \\
\hline & & $\begin{array}{c}T_{\max } \\
\text { (h) }\end{array}$ & $\begin{array}{c}C_{\mathrm{p}_{\max }} \\
(\mu \mathrm{g} / \mathrm{mL})\end{array}$ & $\begin{array}{c}\text { AUC } \\
(\mu \mathrm{g} / \mathrm{mL} \cdot \mathrm{h})\end{array}$ \\
\hline \multirow[t]{3}{*}{ Fasted } & $\mathrm{a}$ & $0.25 / 0.20$ & $10.86 / 10.28$ & $28.13 / 29.36$ \\
\hline & $\mathrm{b}$ & $0.50 / 0.50$ & $9.38 / 9.02$ & $28.72 / 28.30$ \\
\hline & $\mathrm{c}$ & $0.08 / 0.22$ & $8.21 / 10.16$ & $26.44 / 29.23$ \\
\hline \multirow[t]{2}{*}{ Mean $\pm \mathrm{SD}$} & & $0.28 \pm 0.21 /$ & $9.47 \pm 1.31 /$ & $27.76 \pm 1.18 /$ \\
\hline & & $0.31 \pm 0.17$ & $9.82 \pm 0.70$ & $28.96 \pm 0.58$ \\
\hline \multirow[t]{3}{*}{ Fed } & a & $0.75 / 0.78$ & $7.91 / 8.28$ & $30.69 / 28.00$ \\
\hline & $\mathrm{b}$ & $2.00 / 3.12$ & $3.44 / 4.02$ & $15.45 / 19.40$ \\
\hline & $\mathrm{c}$ & $3.00 / 3.02$ & $3.73 / 4.16$ & $17.30 / 20.04$ \\
\hline \multirow[t]{2}{*}{ Mean $\pm \mathrm{SD}$} & & $1.92 \pm 1.13 /$ & $5.03 \pm 2.50^{b}$ & $21.15 \pm 8.32 /$ \\
\hline & & $2.31 \pm 1.32$ & $5.49 \pm 2.42^{b}$ & $22.48 \pm 4.79$ \\
\hline
\end{tabular}

${ }^{a}$ Predicted parameters were calculated from the data simulated by the convolution method based on the GI transit-absorption model. $T_{\max }$ and $C_{\mathrm{pmax}}$ were defined as the time to reach the maximum plasma concentration and the maximum plasma concentration, respectively.

${ }^{b} p<0.05$, compared with fasted condition. 
Fasted Condition
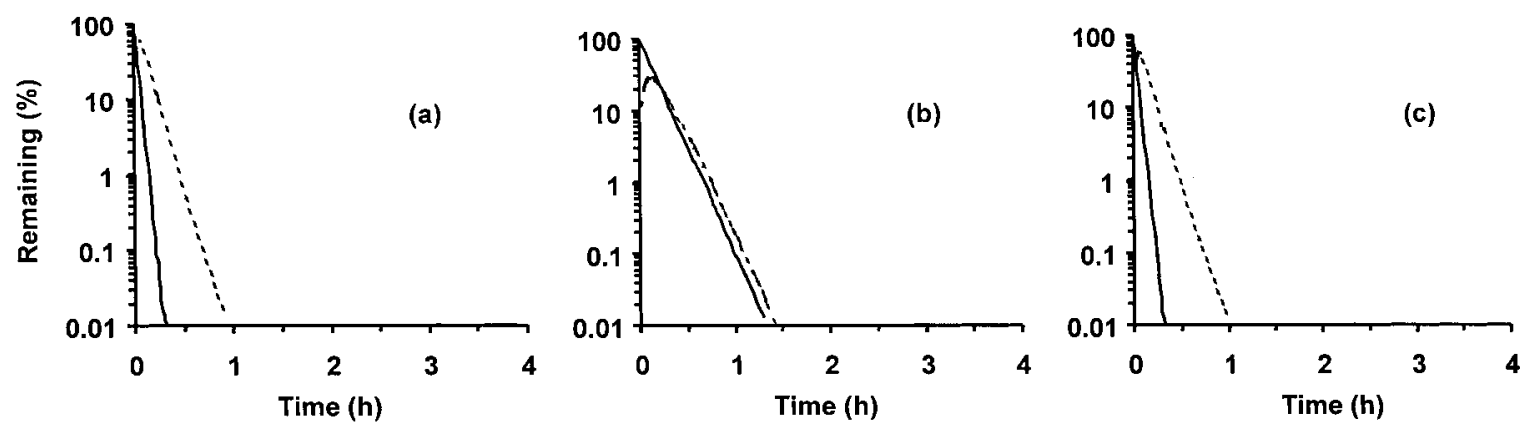

\section{Fed Condition}
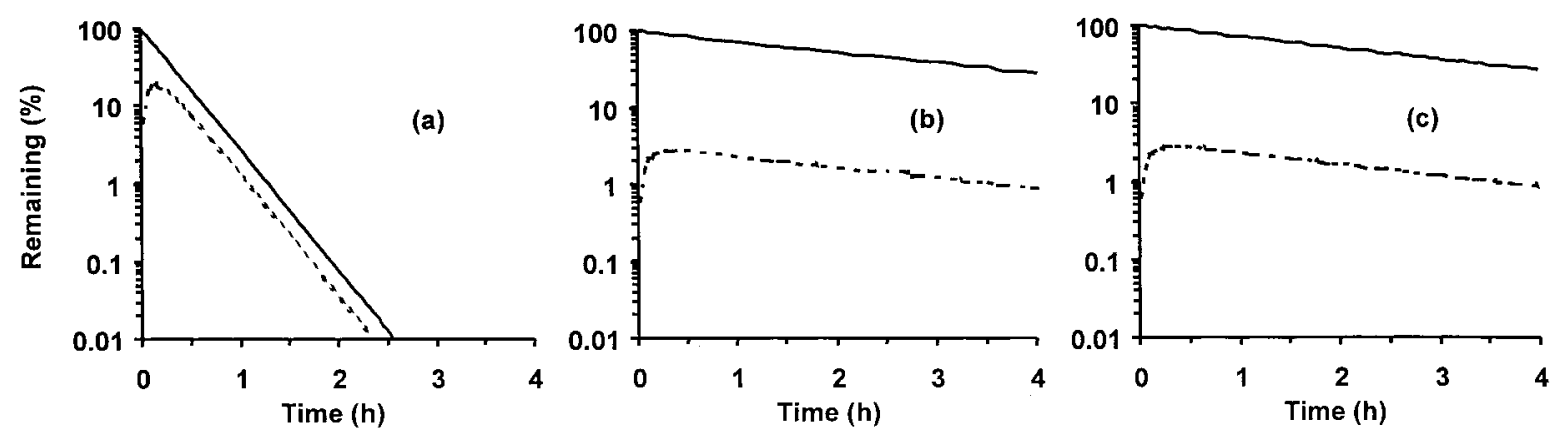

Figure 5. Simulation of theophylline amount remaining in stomach and small intestine after oral administration of aminophylline in each GI transit condition. Time courses of theophylline remaining in stomach and small intestine, which were simulated by the convolution method, are expressed by the solid and dotted lines, respectively.

drug absorption. Figure 5 shows the theophylline-time profiles in the stomach and intestine. This result also indicates that the gastric emptying rate determines the absorption rate of theophylline. However, this relationship is not the case when the value of the gastric emptying rate constant is much larger than the intrinsic absorption rate constant of theophylline, like cases $\mathrm{a}$ and $\mathrm{c}$ in Figure 5. In such a case, the apparent absorption rate constant of theophylline from the GI tract is dependent on not the gastric emptying rate but the intrinsic absorption rate.

In conclusion, the assessment of GI transit for an individual study by gamma scintigraphy and the prediction of the absorption kinetics by the GI transit-absorption model made it possible to investigate the relationship between the GI transit rate and oral drug absorption profile in detail. It was suggested that information about the GI transit for each individual is important to predict the plasma concentration-time curves of orally administered drugs, especially under the fed condition. The combination of gamma scintigraphy and the GI transit-absorption model is a promising method to analyze the absorption kinetics and therefore administer safer and more efficient dosages in human.

\section{REFERENCES}

1. Davis SS, Hardy JG, Fara JW. 1986. Transit of pharmaceutical dosage forms through the small intestine. Gut 27:886-892.

2. Dressman JB, Berardi RR, Elta GH, Gray TM, Montgomery PA, Lau HS, Pelekoudas KL, Szpunar GJ, Wagner JG. 1992. Absorption of flurbiprofen in the fed and fasted state. Pharm Res 9:901-907.

3. Snape WJ, Jr, Battle WM, Schwartz SS, Braunstein SN, Goldstein HA, Alavi A. 1982. Metoclopramide to treat gastroparesis due to diabetes mellitus: A double-blind, controlled trial. Ann Intern Med 96:444-446.

4. Sawamoto T, Haruta S, Kurosaki Y, Higaki K, Kimura T. 1997. Prediction of plasma concentration profile of orally administered drugs on the basis of gastrointestinal transit kinetics and absorbability in rats. J Pharm Pharmacol 49:450-457. 
5. Kutcahi HC. 1998. The gastrointestinal system. In: Berne RM, Levy MN, editors. Physiology, 4th ed. St. Louis: Mosby, p. 589-674.

6. Hebbard GS, Sun WM, Bochner F, Horowitz M. 1995. Pharmacokinetic considerations in gastrointestinal motor disorders. Clin Pharmacokinet 28: 41-66.

7. Casey DL, Beihn RM, Digenis GA, Shabhu MB. 1976. Method for monitoring hard gelatin capsule disintegration times in humans using external scintigraphy. J Pharm Sci 65:1412-1413.

8. Theodorakis MC, Digenis GA, Beihn RM, Shambhu MB, Deland FH. 1980. Rate and pattern of gastric emptying in human using ${ }^{99 \mathrm{~m}}$ Tc-labeled triethylenetetraamine-polystyrene resin. J Pharm Sci 69: 568-571.

9. Digenis GA, Sandefer E. 1991. Gamma scintigraphy and neutron activation techniques in the in vivo assessment of orally administered dosage forms. Crit Rev Ther Drug Carrier Syst 7:309-345.

10. Harris D, Fell JT, Sharma HL, Taylor DC. 1990. GI transit of potential bioadhesive formulations in man: A scintigraphic study. J Controlled Release $12: 45-53$.

11. Christian PE, Moore JG, Sorenson JA, Coleman RE. Welch DM. 1980. Effects of meal size and correction technique on gastric emptying time: Studies with two tracers and opposed detectors. J Nucl Med 9:883-885.

12. Wilding IR, Coupe AJ, Davis SS. 1991. The role of gamma scintigraphy in oral drug delivery. Adv Drug Deliv Revs 7:87-117.

13. Digenis GA, Sandefer EP, Page RC, Doll WJ. 1998. Gamma scintigrapy: An evolving technology in pharmaceutical formulation development-Part 1. Pharm Sci Technol Today 1:100-107.

14. Digenis GA, Sandefer EP, Page RC, Doll WJ. 1998. Gamma scintigrapy: An evolving technology in pharmaceutical formulation development-Part 2. Pharm Sci Technol Today 1:160-165.
15. Palin KJ, Whalley DR, Wilson CG, Davis SS, Phillips AJ. 1982. Determination of gastric-emptying profiles in the rat: Influence of oil structure and volume. Int J Pharm 12:315-322.

16. Goggin JM, Hoskinson JJ, Butine MD, Foster LA, Myers NC. 1998. Scintigraphic assessment of gastric emptying of canned and dry diets in healthy cats. Am J Ver Res 59:388-392.

17. Ogiso T, Iwaki M, Tanino T, Okuyama K, Uno S. 1993. Effect of mexiletine on elimination and metabolic conversion of theophylline and its major metabolites in rats. Biol Pharm Bull 16:163-167.

18. Yano Y, Yamaoka K, Tanaka H. 1988. A nonlinear least squares program, MULTI (FILT), based on fast inverse laplase transform for microcomputer. Chem Pharm Bull 37:1035-1038.

19. Kakemi K, Sezaki H, Konishi R, Kimura T, Okita A. 1970. Effect of bile salts on the gastrointestinal absorption of drugs.II. Mechanism of the enhancement of the intestinal absorption of sulfaguanidine by bile salts. Chem Pharm Bull 18:1034-1039.

20. Haruta S, Iwasaki N, Ogawara K, Higaki K, Kimura T. 1998. Absorption behavior of orally administered drugs in rats treated with propantheline. J Pharm Sci 87:1081-1085.

21. Shiu GK, Sager AO, Velagapudi RB, Prasad VK, Skelly JP. 1988. The effect of food on the absorption of controlled-release theophylline in mini-swine. Pharm Res 5:48-52.

22. Davis SS, Hardy JG, Fara JW. 1986. Transit of pharmaceutical dosage forms through the small intestine. Gut 27:886-892.

23. Peh KK, Yuen KH. 1996. Indirect gastrointestinla transit monitoring and absorption of theophylline. Int J Pharm 139:95-103.

24. Abrahamsson B, Alpsten M, Hugosson M, Jonsson UE, Sundgren M, Svenheden A, Tolli J. 1993. Absorption, gastrointestinal transit, and tablet erosion of felodipine extended-release (ER) tablets. Pharm Res 10:709-714. 